\title{
Synchronizing a 2D continuum of two populations of neural masses Olivier Faugeras ${ }^{* 1}$ and Jean-Jacques Slotine ${ }^{2}$
}

\author{
Address: ${ }^{1}$ Odyssee Laboratory, INRIA/ENPC/ENS, INRIA Sophia Antipolis, France and ${ }^{2}$ Nonlinear Systems Laboratory, MIT, Cambridge, MA, USA \\ Email: Olivier Faugeras* - olivier.faugeras@sophia.inria.fr; Jean-Jacques Slotine - jjs@mit.edu \\ * Corresponding author
}

from Sixteenth Annual Computational Neuroscience Meeting: CNS*2007

Toronto, Canada. 7-12 July 2007

Published: 6 July 2007

BMC Neuroscience 2007, 8(Suppl 2):PI78 doi:I0.I I86/I47I-2202-8-S2-PI78

(c) 2007 Faugeras and Slotine; licensee BioMed Central Ltd.

\begin{abstract}
Background
Neural field models of firing rate activity have played a major role in developing an understanding of the dynamics of neural tissues [1]. In this paper we study the possibility of synchronizing a two-dimensional neural field of excitatory and inhibitory layers of neural masses. This is the first step toward an investigation of the properties of visual areas in man and monkey. Each population is described by its post-synaptic potential (PSP), hence the state space is a two-dimensional function defined on the $2 \mathrm{D}$ continuum. The field is modeled by an integro-differential equation. At a given point in the continuum this equation models the synaptic integration of the neural mass through a linear term and the contributions of its neighbors to the variation of its PSP through a spatial integration of their firing rates weighted by a connectivity function. The firing rates are classically related to the PSPs through sigmoidal functions.
\end{abstract}

\section{Methods}

We use techniques from functional analysis to establish a sufficient condition for the neural masses in the continuum to globally synchronize. The Frechet derivative of the right-hand side of the integro-differential equation is shown to define a compact operator on the set of square integrable functions. The sufficient condition described below is obtained by imposing that the spectrum of the symmetric part of this operator be negative.

\section{Results}

We provide sufficient conditions on the connectivity matrix of the neural field for the existence of an homogeneous solution. We perform a classical linear stability analysis of this solution in this multidimensional framework [2]. We then use an extension of the contraction analysis for nonlinear systems [3] and of the analysis of concurrent synchronization in dynamic system networks [4] to obtain sufficient conditions for the neural masses in the continuum to globally synchronize when they receive the same input. In the case where the connectivity matrix of the network is translation invariant the condition can be elegantly expressed in terms of its Fourier transform. We also show that this condition implies the linear stability of the homogeneous solution.

\section{Conclusion}

The sufficient condition described above raises interesting biological questions that may be partially answered through such measurement techniques as Optical Imaging (OI). Conversely, OI measurements can provide clues for the spatial shape of the connectivity matrix. Finally these results open the door to a principled investigation of the properties of the visual areas in man and monkey where spatial synchronization would be the support of spatial similarity in terms of such visual features as color, texture, edges, optical flow.

\section{References}

I. Coombes S: Waves, bumps, and patterns in neural field theories. Biol Cybern 2005, 93:91-108. 
2. Gerstner W, Kistler W: Spiking Neuron Models. Cambridge University Press; 2002.

3. Lohmiller W, Slotine JJE: Contraction analysis for nonlinear systems. Automatica 1998, 34:6.

4. Pham Q-C, Slotine JJE: Stable concurrent synchronization in dynamic system networks. Neural Networks 2007, 20:I.

Publish with Biomed Central and every scientist can read your work free of charge

"BioMed Central will be the most significant development for disseminating the results of biomedical research in our lifetime. " Sir Paul Nurse, Cancer Research UK

Your research papers will be:

- available free of charge to the entire biomedical community

- peer reviewed and published immediately upon acceptance

- cited in PubMed and archived on PubMed Central

- yours - you keep the copyright 